

\title{
RobotGreen: A Teleoperated Agricultural Robot for Structured environments
}

\author{
César Peña ${ }^{1,}$, Cristhian Riaño ${ }^{1,2}$ and Gonzalo Moreno ${ }^{1}$ \\ ${ }^{1}$ Department of Mechanical, Mechatronic and Industrial Engineering, University of Pamplona, Km 1 via Bucaramanga, 543050, \\ Pamplona, Colombia. \\ ${ }^{2}$ University Campus Darcy Ribeiro/ Asa Norte, 04386, Brasilia, Brasil.
}

Received 1 October 2018; Accepted 17 February 2019

\begin{abstract}
This paper presents the development of a remote robotic platform to monitor and take care of urban crops. The agricultural robot performs activities such as sowing, irrigation, fumigation, and pruning activities on a small scalable structured crop. The generalities of the design of an anthropomorphic robot with five degrees of freedom, the description of the development of a multi-purpose end effector for the development of agricultural tasks, the implemented architecture of the teleoperation system of the robot is presented, including a visual support system and the employment of a low-cost master robot that allows the slave robot to be controlled more intuitively. Finally, some experimental results that show the functioning of the system are presented.
\end{abstract}

Keywords: Robot, agriculture, teleoperation, urban, remote sensors, eco-friendly design.

\section{Introduction}

With the passage of time humanity is becoming aware of the preservation of natural resources. In retrospect, many cases in which industrial processes prevailed even over harmful environmental effects could be noted. Nowadays man has realized that natural resources are very limited and that it is necessary to comply with a series of rules to protect the environment. In this new wave of conservationism and sustainability, several companies have proposed a large number of alternatives in favor of the protection of the environment. Some of them are the use of renewable energies on a large scale, clean or environmentally friendly industrial production [1], use of recyclable or natural materials, minimization and waste management, the proper storage or disposal of waste, among others.

Although large and medium-sized industries generate large environmental impacts [2], the need to promote the same culture spreads to small businesses and even to the population in general. Following this last idea it is conceivable to see how in recent years ideas such as energy generation on a domestic scale, architectural designs that take advantage of thermodynamic effects [3], collection of rainwater for toilets $[4,5]$, green roofs [6], vertical crops in buildings $[7,8]$, among many others have emerged.

These latest applications demonstrate how humanity is radically changing the conception that industrial developments and technology go against the environment, these on the contrary could guarantee the care of it, making them sustainable. A good example is the incorporation of new technologies such as robots in agricultural activities performing a wide variety of tasks [9]. These activities include: fruit harvesting [10], monitoring [11], loading and

*E-mail address: cesarapc@unipamplona.edu.co ISSN: 1791-2377 @ 2019 Eastern Macedonia and Thrace Institute of Technology. All rights reserved. doi:10.25103/jestr.121.10 unloading of agricultural material [12], transport assistance for harvested fruits [13], irrigation [14], fumigation, fertilization [15], weeding, automatic grafting, plowing [16], and sowing [17].

For the development of these activities, the agricultural robots have been equipped with advanced features compared to those of other robots, to guarantee their efficiency and effectiveness. e.g. for the collection of delicate fruits there are robots with deformable clamps [18], they have been equipped with the ability to interact and cooperate with other robots [19], $\mathrm{pH}$ measurement, humidity, automatic location of plants [20], wide range navigation [21, 22], guided by vision to avoid obstacles [23], land surveys for its displacement [24], some can even be reconfigured to achieve complex manipulations [25], others implement redundancy strategies [26], to perform tasks too complex or where you want a robot to do the work of a man to avoid exposure to hazardous environments as in the case of fumigation, teleoperated robots are implemented [15].

This project is aimed at the development of small structured crops or for interiors. The general idea is that citizens may have small crops in their own homes for the production of some types of food or infusions. Since the general population is used to doing a lot of work, they cannot afford to go constantly to their homes, which is why it is proposed that these crops be monitored or tended remotely by means of a robot, which will be responsible for carrying out the main tasks.

This teleoperation can be developed on different levels, which range from a supervised control using a smart phone to perform basic and repetitive tasks such as irrigation, to an advanced teleoperation that requires the use of a master robot (MR) for the development of complex tasks such as planting. This last case is contemplated when an urban crop is located on a roof, a terrace, or in a vertical garden in order to keep users from having to go to them avoiding possible accidents. 
This article is organized as follows: in Section 2; the technological development is presented, in it; the generalities of the robot's design are described, it is then followed by the architecture of teleoperation systems; the results are analyzed and discussed in Section 3, and finally, the conclusions are presented in Section 4.

\section{Technological Development}

Generalities of Design of the Robotic Structure: For the study phase and design of the agricultural robot, the methodology of Quality Function Developed (QFD) is used as a method to define the development of its activities. This methodology compiles the requirements and needs of the robot and displays them in a set of technical and operative characteristics conducive to creating a concept that meets them. The methodology, rather than providing quantitative data, provides information of a fundamental qualitative nature to generate the design concept and explore solutions that adapt to the tools available for the execution of this project [27].

The requirements of the project represent the needs to be solved with the manufacturing of the robot. Some needs demand a higher level of attention, therefore; the degree of importance for each project requirement must be established correctly. The Mudge diagram is a tool that allows the comparison of project requirements aiming to generate a hierarchy among them according to the degree of importance and relevance in the project. The comparison in the Mudge diagram is done by attributing a value in each evaluation of two functions. The scale used to define the degree of importance considering values between 1 and 5 , where 5 is the highest and 1 the lowest degree of importance. These values are represented by the letters A, B, C, D, E with respective values of $5,4,3,2,1$.

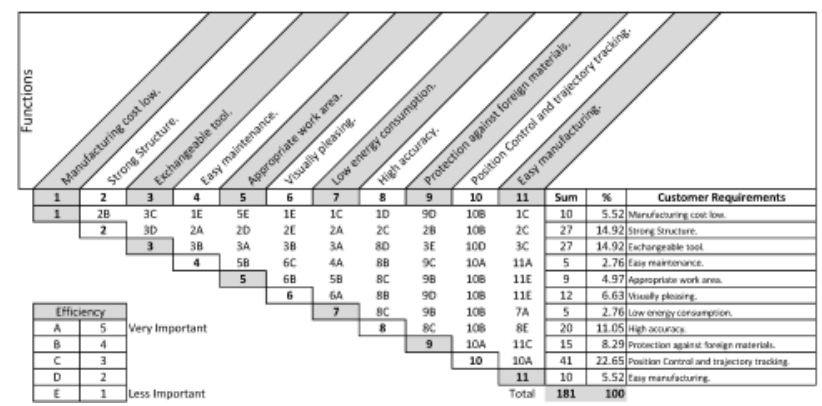

Fig. 1. Mudge Diagram for Agricultural Indoor Robot

In Fig. 1 the result of applying the Mudge diagram is presented. The scale and degree of importance that define the relevance of the requirements in the project can be appreciated [28].

The requirements of the project derived from the needs after applying the Mudge diagram are presented in Fig. 2 through the Pareto diagram. The bars represent each of the requirements defined for the robot with the value resulting from the comparison with other requirements. The curve indicates accumulations that go up to the value of 100 percent. An analysis of the diagram allows us to deduce that if efforts and resources are focused on satisfying the first 6 requirements, the project approaches the solution 60 percent for the needs raised by the users of the agricultural robot.

The solution concept obtained for the requirements mentioned in the agricultural robot project establishes the best solution for the robotic configuration in that of an anthropomorphic structure. Compared to closed chain robotic configurations, this arrangement provides greater work area, and a high degree of maneuverability. With the right selection of motors, precision, torque and correct operation of the position and trajectory control are guaranteed.

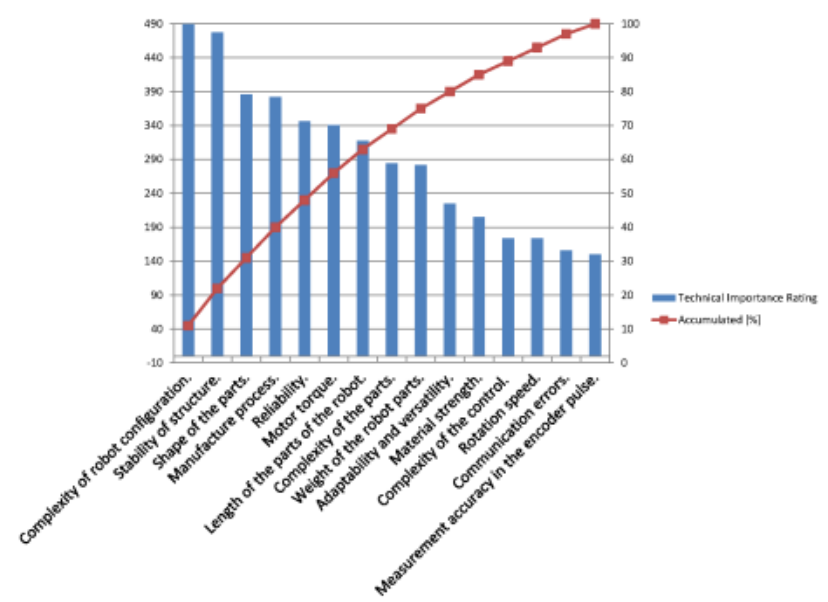

Fig. 2: Pareto diagram of the robot for agricultural applications.

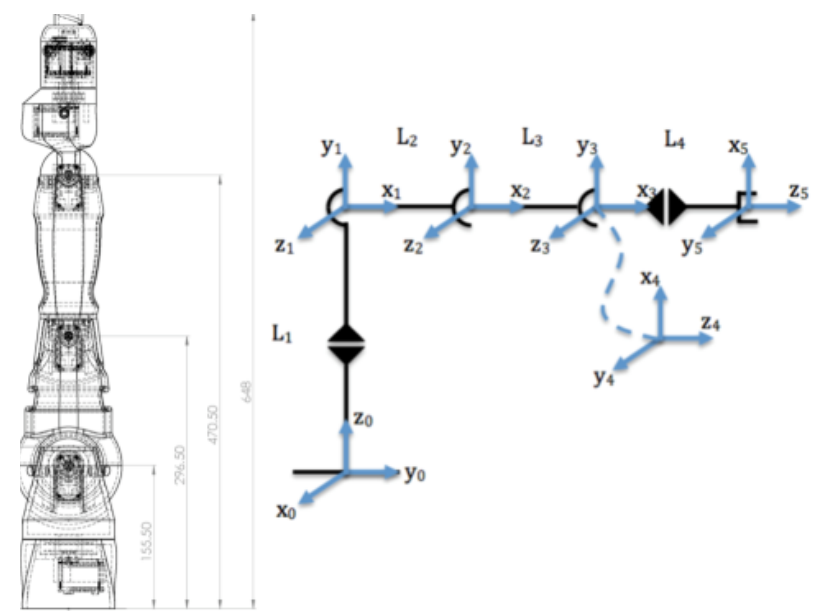

(a). Dimensioning.

(b). Cinematic scheme

Fig. 3. Anthropomorphic robot structure with $5 \mathrm{DF}$.

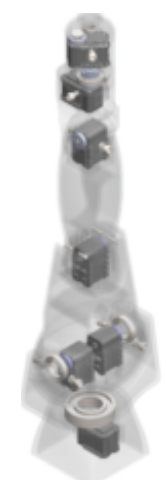

(a). Arrangement of the drives.

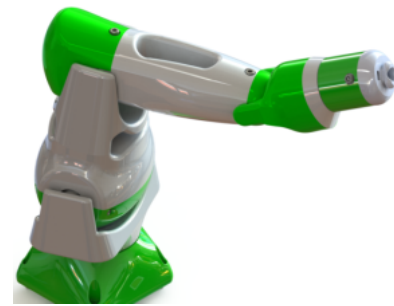

(b). Robot
Fig. 4. Design of Anthropomorphic robot with 5 DoF.

In Figs. 3 and 4 the design concepts represented by a virtual prototype modeled in CAD software are presented, containing the solution displayed in detail. With the virtual prototype data such as physical properties, movement analysis, verification of constraints, are estimated as well as other analysis that allow evaluation of the design concept. 
After studying the virtual prototype and making the necessary adjustments, the construction and assembly route of the robot is generated and adjusted to the design guidelines obtained.

Once the type of structure was proposed, the kinematic model of the robot was made by the traditional method of Denavit-Hartenberg, as well as by the successive displacement of screws using the Rodrigues equation [29] in its denotation of homogeneous transformation matrix for each joint $\left(\boldsymbol{A}_{\boldsymbol{i}}\right)$, to find the closing equation of the end effector with respect to the origin $\left(\boldsymbol{A}_{\boldsymbol{e}}\right)$ given its initial position $\left(\boldsymbol{A}_{\boldsymbol{e} 0}\right)$. In the latter case, the screw axes $\left(\boldsymbol{s}_{\boldsymbol{i}}\right)$ coincide with the $\boldsymbol{Z}_{\boldsymbol{i}-\mathbf{1}}$ (with $i$ from 1 to 5 ) and the normal vector from the origin to the screw axis is denoted as $\boldsymbol{s}_{\mathbf{0}}$.

$A_{e}=A_{1} A_{2} A_{3} A_{4} A_{5} A_{e 0}=A_{h} A_{e 0}$

The conventional Jacobian matrix is obtained by horizontally concatenating the vectors $\mathrm{J}_{\mathrm{i}}$ defined by the Eq. (2) for $i$ from 1 to 5 (since all the joints of the robot are rotational), where.$^{i-1} p_{n}$ are the vectors of the articulation system unto the end effector of the robot.

$\mathbf{J}_{i}=\left[\begin{array}{c}{ }^{0} \mathbf{Z}_{i-1} \times{ }^{i-1} \mathbf{p}_{n} \\ { }^{0} \mathbf{Z}_{i-1}\end{array}\right]$

This robot basically has two types of singularities: those of the outer limits $\left(\mathrm{q}_{3}=0\right.$, see section 2.3 and Fig. 17) and the internal ones that occur when the end effector is on the $Z_{0}$ axis (coordinates $x_{5}$ and $y_{5}$ null). To solve this second singularity in terms of inverse kinematics, a choice was made to keep a record of the joint coordinate $\mathrm{q}_{1}$ (memory) and take the last one when passing through a singular point. In regards to the velocity level, the left-side pseudo-reverse method is implemented minimizing the quadratic error $\left(\mathrm{J}_{\mathrm{i}}^{+}=\left[\mathrm{J}^{\mathrm{t}} \mathrm{J}\right]^{-1} \mathrm{~J}^{\mathrm{t}}\right)$, another alternative is to use the Singularity Isolation plus Compact QP (SICQP) method described in [30].

In Fig. 4 the arrangement of the drives in the robot are illustrated, in it, it can be noted that the second joint has two motors in parallel position given that these are ones that must exert a greater torque. For the selection of the motors, the inverse dynamic model of the robot was estimated by means of the Lagrangian formulation, see Eqs. (3) and (4)

$\frac{d}{d t} \frac{\partial L}{\partial \dot{q}_{i}}-\frac{\partial L}{\partial q_{i}}=\tau$

$$
L=E_{c}-E_{p}
$$

where $q_{i}$ are the generalized or articulate coordinates, $\tau_{\bullet}$ is the applied pair vectors, $L$ is the Lagrangian function, $E_{c} \bullet$ is the kinetic energy, and $E_{p}$ is the potential energy.

When replacing the masses, inertias, frictions and dimensional parameters of the robot, the expressed model equation was obtained an expressed as follows:

$$
\tau=\mathbf{D}(q) \ddot{q}+\mathbf{H}(q, \dot{q})+\mathbf{C}(q)+\mathbf{F}_{\mathbf{v}} \dot{q}
$$

where $\mathrm{D}$ is the inertial matrix, $\mathrm{H}$ is the vector of coriolis and centrifugal forces, $\mathrm{C}$ is the vector of gravity force, and $F_{v}$ is the robot's friction coefficient matrix.



Fig. 5. Simulation for the calculation of the maximum pairs of the anthropomorphic robot.

Subsequently, with the inverse dynamic model (see Fig. 5), a simulation of the robot was made, placing it in the most demanding configuration and applying to each of the joints a trapezoidal trajectory according to the parameters of acceleration times and maximum speed required. In this way the data was obtained to select the appropriate motors.

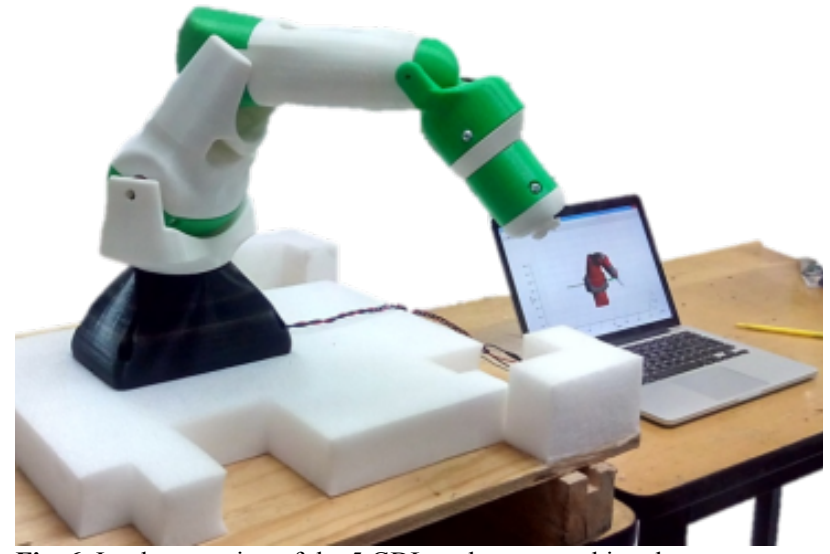

Fig. 6. Implementation of the 5 GDL anthropomorphic robot.

In Fig. 6, the manufactured and assembled robot is illustrated. This robot was built through a $3 \mathrm{D}$ printer using Lactic Polyacid (LPA) as material since it is a totally biodegradable polymer with excellent mechanical characteristics.

For the development of agricultural tasks the robot is equipped with a multifunctional tool that is coupled to the motorized system and consists of the following functions:

Shovel to excavate or remove material in the crop.

Clamps to manipulate or move an element.

Cutting element that operates as crop scissors.

Irrigation instrument with the possibility of spraying, supplying.

In Fig. 7 it can be seen how the end effector has a tool in the form of a shovel, which can be divided in half allowing its parts to come together or separate. This feature will allow you to act as grippers in addition to a removal element. Note that for this case two protuberances were designed in the form of fingers in the upper part in order to improve the grip of objects. 


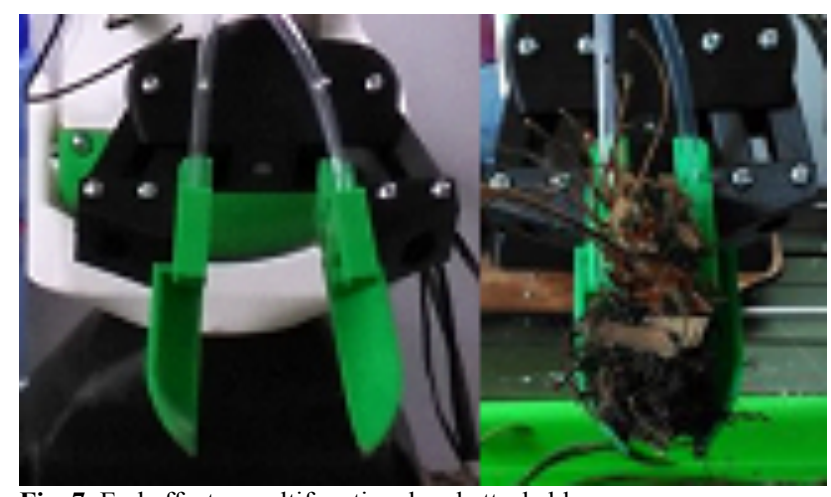

Fig. 7. End effector multifunctional and attachable.

Another characteristic is that the point of union of these two parts (at the end) is thin and sharp which can be used to perform cutting operations of small stems or roots. Additionally, it has two ducts where the water supply and fertilizer systems are incorporated. If irrigation operations are desired (it could also be used for fumigation when a dispersing element is coupled). It must be stressed that the robot has the ability to easily attach various tools which will allow you to expand its applications.

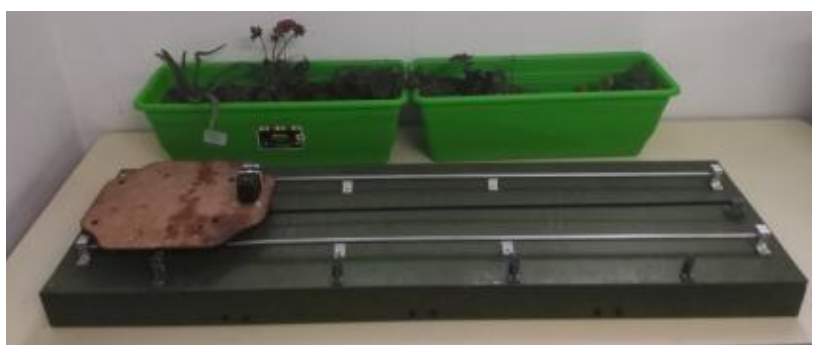

Fig. 8. Translation mechanism for the robot. Architecture of the teleoperation system

To satisfy the requirement of the project that sets the working space of the robot as important, the solution concept defines an additional mechanism that allows the robot to be moved in one direction. The mechanism is a motorized rail system which can be modified to reach different distances and allow the inclusion of new crop sections [31]. In Fig. 8 a photograph of the device can be seen.

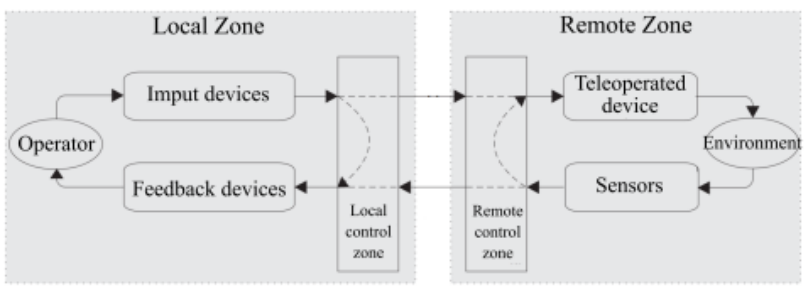

Fig. 9. Teleoperation system architecture

The system can be divided into two zones: local and remote. The first is where the user or operator is located, which through the use of input devices (keyboard, mouse, master device, and smart phone) generates directives or commands to operate the system. The user in turn has feedback devices which allow him to handle information related to the remote environment; in this case he has a screen that displays the images captured by a camera in the remote environment, as well as information coming from sensors. Since a master device was implemented, a controller was required in the local area which is responsible for controlling the actuators of this device, when a particular force is required to be executed. In Fig. 9 we can see the general architecture of the teleoperation system implemented for remote cultivation.

In the remote area the devices to be controlled can be found, which in this case are: the agricultural robot and the displacement rail, the control box can be a PC, the environment is composed by the crop, the sensors including the electronic devices involved and the communications system.

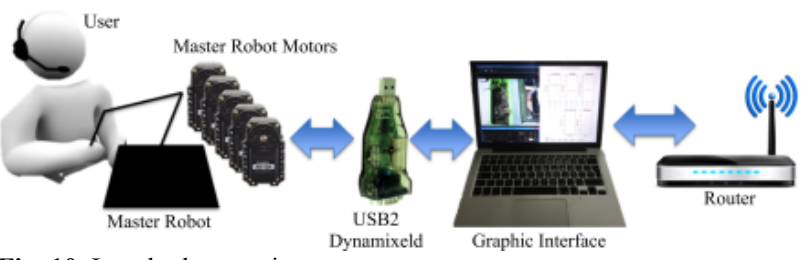

Fig. 10. Local teleoperation system zone

In Fig. 10 the elements that make up the local area of the teleoperation system can be seen. The user has a computer that illustrates the information captured by the sensors in the remote environment. In turn, the user can enter the operation commands in various ways. You can use the mouse to press buttons in a graphical interface (quite intuitive) with specific commands such as the watering of a certain plant, the change of position of the end effector of the robot on the axes $x, y, z$, changes of orientations, among others. If you open the remote console application you can write and execute textual commands (higher level of platform control). And finally it has an operation mode based on a haptic device (master robot) that has a homothetic structure of the agricultural robot, by moving this device the remote robot will follow them. The control system is implemented in the computer, which then communicates via User Datagram Protocol (UDP) through a router with the remote area.

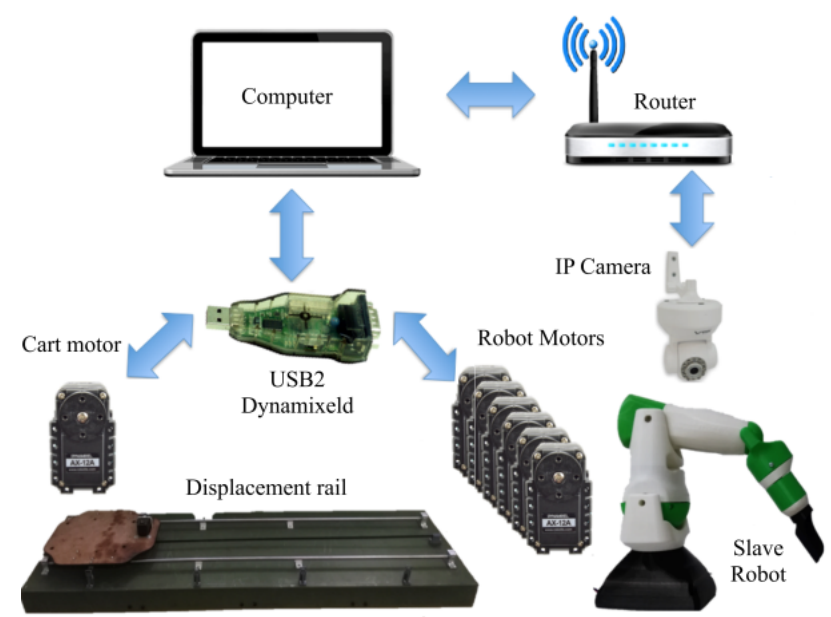

Fig. 11. Remote teleoperation system zone

As for the remote area, it was divided into two parts in order to better illustrate its components. The first part consists of the agricultural robot which has 4 AX12 motors, 3 high torque MX64 motors, a displacement rail that significantly increases the working space of the robot which is driven by an AX12 motor. Since these two devices have actuators from the company Dynamixel, a USB2 Dynamixel card is used for its operation, which is commanded by means of a computer, as can be seen in Fig. 11.

It can also be seen in this figure that there is an IP camera that communicates via WIFI or Ethernet to perform a visual feedback of the remote area. Optionally, the robot can 
have a high resolution web camera in its end effector in case it needs to visualize more clearly any object in the environment.

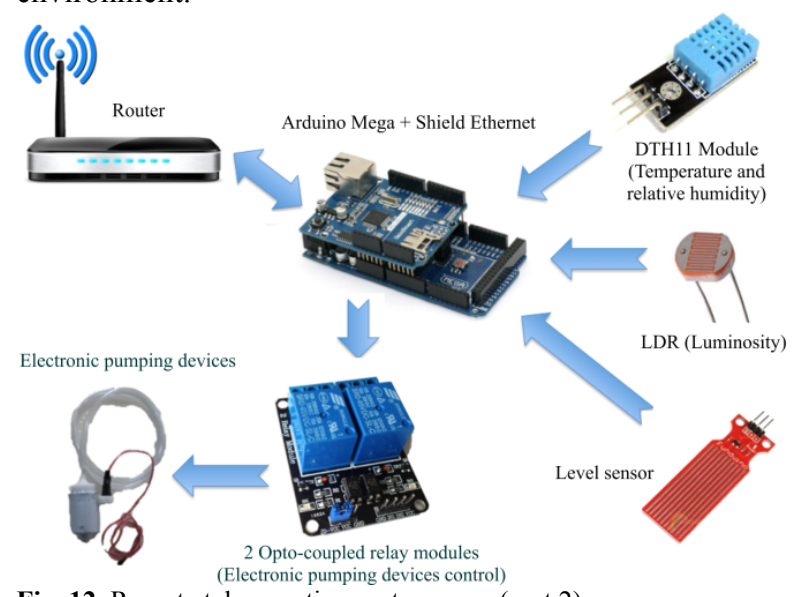

Fig. 12. Remote teleoperation system zone (part 2).

The second part of the remote zone (see Fig. 12) is composed of an Arduino Mega card equipped with an Ethernet Shield that allows you to send and receive information via UDP, a digital temperature humidity sensor (DTH11) that allows you to estimate the temperature of the environment and relative humidity, a light sensor or light dependent resistor (LDR), two level sensors to know the state of the liquids in the water and fertilizer storage tanks (or fumigation liquid) and a module of two optocoupled relays that control the drive of the electronic pumping devices [31].

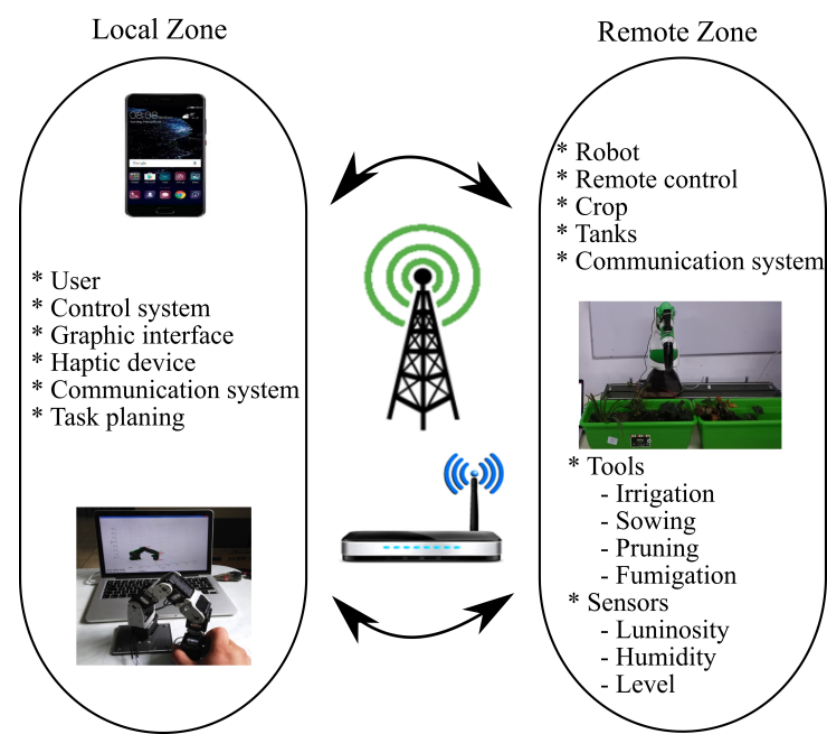

Fig. 13. General diagram and summary of the system's architecture.

In Fig. 13 we can see a general and summarized diagram of the architecture of the system. It should be noted that the communications channel was proposed for a local area network, however it can be easily expanded to the Internet in order to increase the distance between the local and remote area. Since the universal protocol of datagrams was used for the sending of commands, the possibility of generating mobile applications for the control of the agricultural platform from a smartphone or a tablet is open.

Preliminary Approaches for the Teleoperatrion of the Robotic Arm: Moving the robot by specifying the joint coordinates can be an uncomfortable task for the user, because the new location (position and orientation) of the robot end effector cannot be calculated intuitively (calculations corresponding to the direct kinematics of the robot). On the other hand, specifying a new reference location for the robot is not as trivial a task as thought given that the robot has only 5 degrees of freedom having some limitations related to movement. In the same way there are some mechanical and electrical restrictions that prevent the robot from moving to a large number of locations. In order to solve this problem, a kinematic study of the working space of the robot is carried out and a series of aids are generated to illustrate the user about the allowed movements that the robot can make.



Fig. 14. Inner limit of the robot's wrist workspace without taking collisions into account.

The robot's workspace corresponds to the points at which its end effector can be accessed. It must be taken into consideration that although the robot is capable of reaching a certain position, this does not mean that it may be able to do it with any kind of orientation. For this reason, contemplating which points are those in which the robot's wrist can access should be the first thing to do (remember that the wrist is in charge of changing the orientation of the end effector). The point of the robot's wrist corresponds to the origin of system 3, which can be seen in Fig. 14.

The blue sphere corresponds to the inner limit that the robot's wrist can reach. This sphere's center is the robot's shoulder (System 1) and has as radius the distance between the origins of the systems 3 and 1 when joint 2 takes the value of 90 degrees (maximum mechanical limit of this articulation defined by the geometries of the links 2 and 3 of the robot).

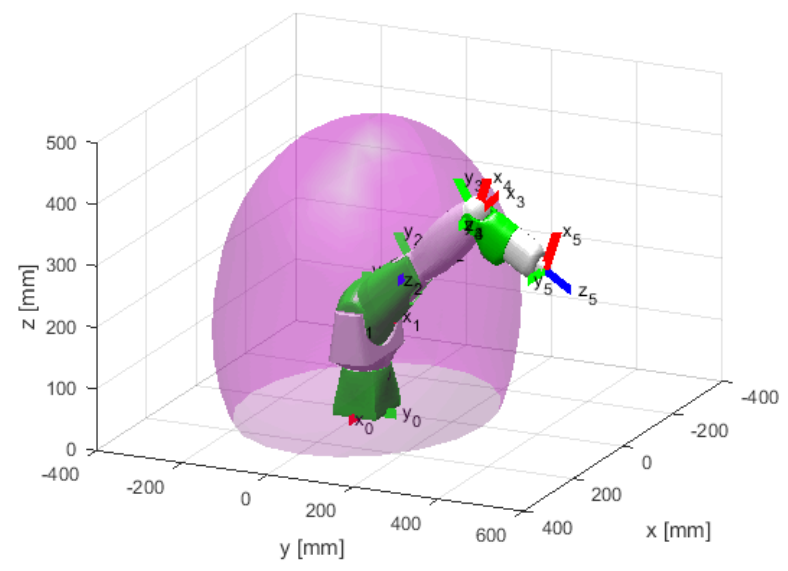

Fig. 15. Outer limit of the workspace of the robot's wrist. 
In an analogous way, the outer limit of the workspace of the robot's wrist is calculated. It can be seen that it is coincident when links 2 and 3 are aligned. In Fig. 15 the robot is illustrated in this type of configuration.

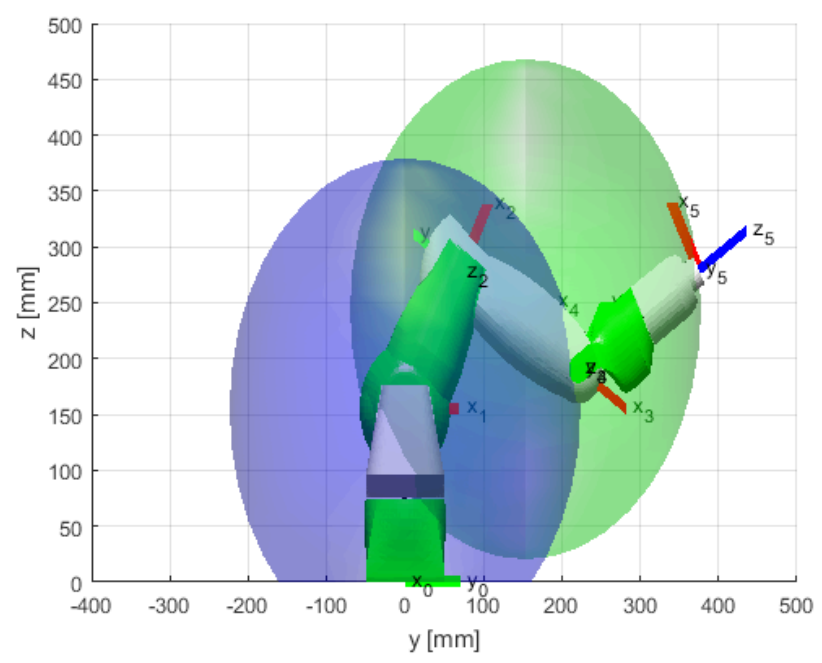

Fig. 16. Inner limit of the workspace of the robot's end effector.

In order to make the operation of the robot more intuitive, the workspace of the end effector is calculated from its current orientation. The cross section of this space corresponds to the same as the wrist but displaced to a distance equivalent to the difference between the positions of the systems' coordinates 4 and 5, as shown in Fig. 16.



Fig. 17. Outer limit of the workspace of the robot's end effector

In an analogous manner, the relationship between the outer limit of the working space of the wrist and the end effector is illustrated in Fig. 17. It should be noted that these workspaces revolve around the $z_{0}$ axis according to joint position number one. From these preliminary approaches, the operating ranges of the robot end effector can be established. Additionally the robot's designed kinematic control system restricts the boundary angles of each of the joints to prevent the links from colliding with each other.

The teleoperation of the agricultural robot can be performed by the user using 4 different modes:

- Trajectory planning using an isochronous mode.

- Movement in the task space mode.

Tracking of a master robot mode.

Pre-loaded routines mode

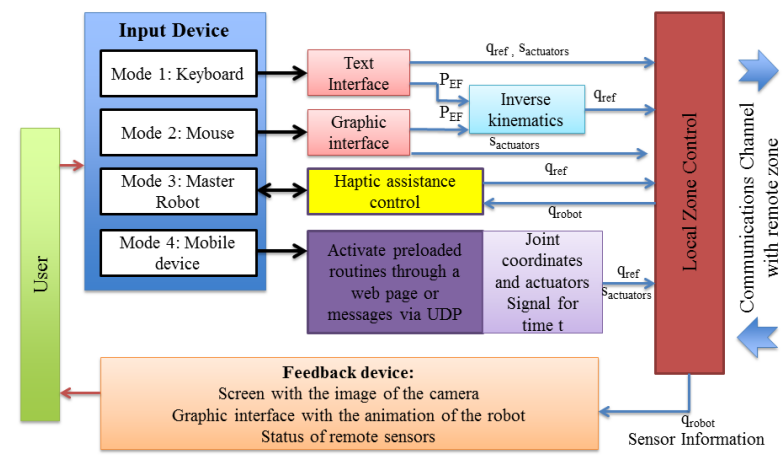

Fig. 18. Local zone control scheme of the teleoperation system

Fig. 18 shows the local zone control scheme of the system including the input devices that the user can use in each of the teleoperation modes of the robot. In this figure it can be seen that the user may use: a keyboard to send preset commands (e.g. go to home position or go to a specific joint coordinates), a mouse to interact with a graphical interface by moving the robot end effector (movement in the space of the task), a master robot (Tracking Mode) or a mobile device (cell phone, tablet, laptop) to activate preloaded routines from a web page.

Trajectory Planning Using an Isochronous Mode: In this type of trajectory, all joints start and finish at the same time.

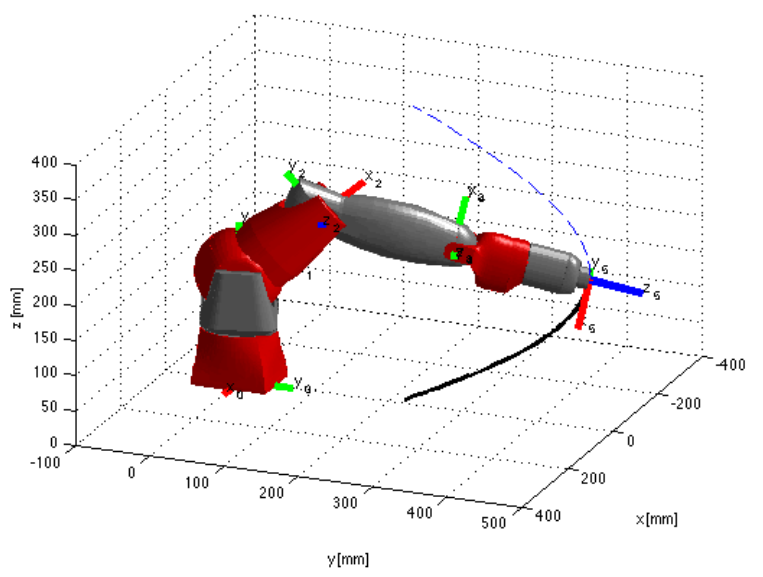

Fig. 19. Robot developing an isochronous trajectory

In Fig. 19 the three-dimensional representation of the execution of an example of this type of trajectories is illustrated. It must be taken into account that the profile implemented in each of the joints is a trapezoidal type.

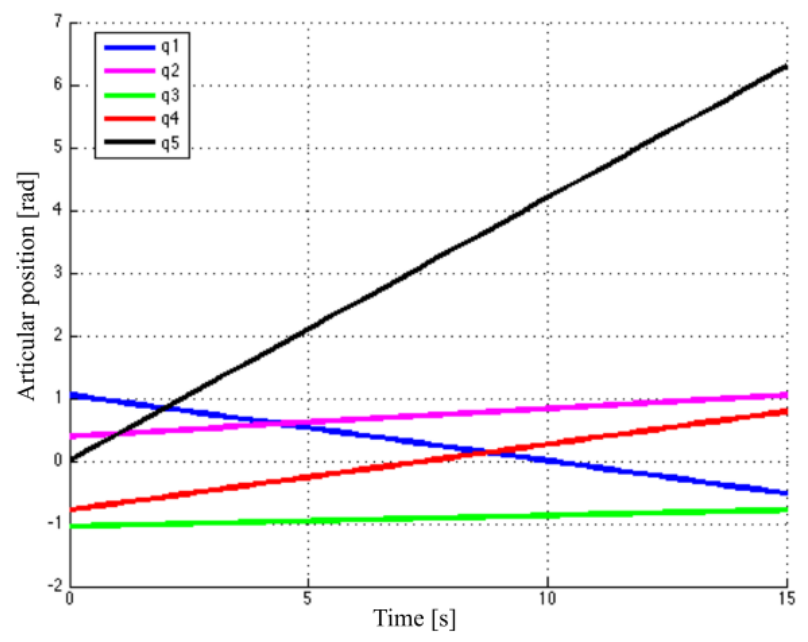


Fig. 20. Evolution of the joint coordinates of the robot developing an isochronous path

As can be seen in Fig. 20 all the joints begin to move at the same time and finish at the same moment.

Teleoperation of the Robot Using the Movement in the Task Space Mode: This mode of teleoperation is based on the operator being able to change the position of the end effector according to the coordinate axes $x, y$ and $z$ at the base of the robot as well as specifying the slope of the end effector with respect to the $X Y$ plane of the zero system, this slope is controlled by the fourth degree of freedom.

Given that there are several mechanical restrictions that prevent the robot from moving in some directions, the need to provide the user with information on the robot's current position and the movements it can make from it was detected, evidencing the limitations.

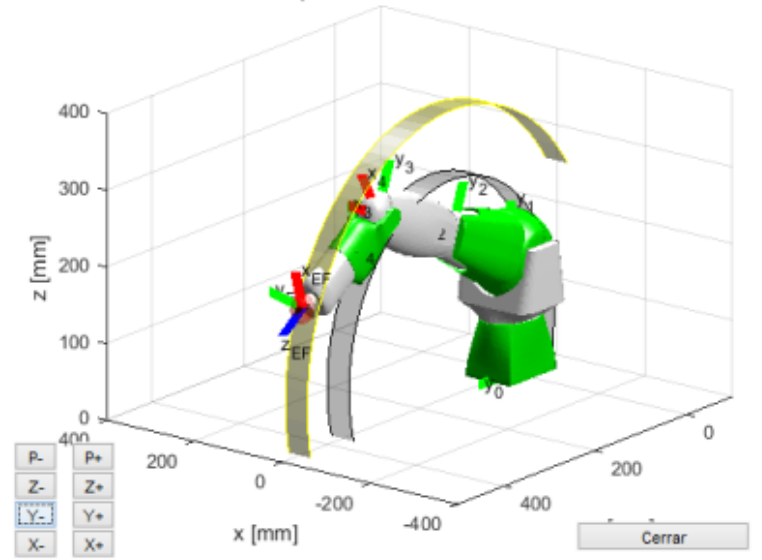

Fig. 21. Indication of arrival at the outer limit of the workspace

To provide a better guide to the operator of the teleoperated system, visual signals are generated to indicate when the robot reaches one of its limits or how close it is to them. For example, in Fig. 21 the inner and outer limits of the workspace allowed for the robot are illustrated. In the case that the reference indicated by the user reaches one of its workspace limits, this limit will change color informing the user of this event.

Even with guide signals, it is very common for the user to generate references that the robot is not able to achieve, which is why an algorithm that detects whether or not the robot reaches the reference was generated, if it does not reach it, it calculates a solution that is viable for the robot and executes it. This allows a more comfortable handling of the robot without generating interruptions.

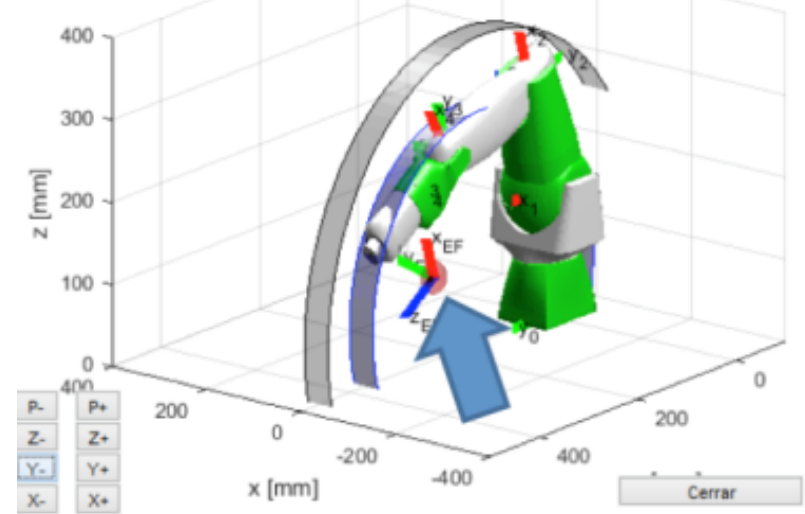

Fig. 22. Reference exceeding the inner limit of the workspace.
In Fig. 22 this case can be seen, where the solidarity sphere coordinated with the end effector system indicate the reference or objective proposed by the user for the robot end effector.

Teleoperation of the Robot Using the Tracking of a Master Robot Mode: Despite the visual indications provided to the user, teleoperation of the robot is not very intuitive for some of them using the mouse and / or the keyboard as input devices. For this reason, the use of master devices that facilitate the establishment of references was proposed, however, as is well known, this type of devices are of considerable cost, so the design and implementation of a device using the same type of robot servomotors as actuators (AX12 motor) was proceeded since they are relatively low cost bearing in mind the great benefits they provide.

The general idea was to develop a device that reflects the user guiding forces allowing him to estimate in an intuitive way when the links of the real robot are close to colliding in order to avoid sending erroneous references or crashes of the robot.

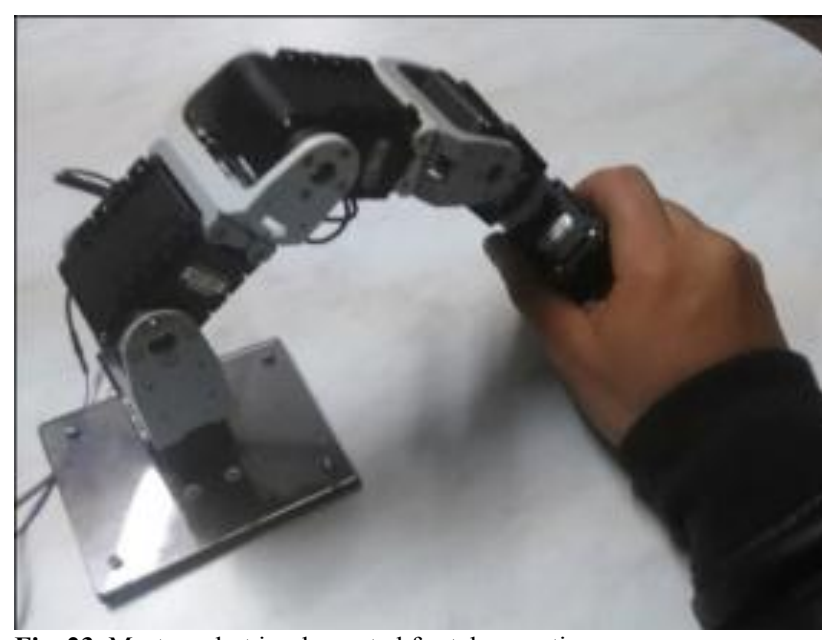

Fig. 23. Master robot implemented for teleoperation.

The developed device offers the user comfort to reflect a homothetic behavior to the anthropomorphic structure of the slave robot (SR) (agricultural robot), see Fig. 23. This implies that the user does not need to know by means of visual signals the limits of the workspace of the robot end effector, you just have to guide the end of the MR with your hand and the SR will follow the established reference.

Since the two robots have very similar kinematic constraints, they allow the user to easily interpret the logic of SR movements. For this reason it is not necessary to perform any kinematic transformation between them (only scaling). Although the movement ranges of the joints of the MR are greater than those of the SR, they were limited via software to make them coincide fully.

The use of this device makes the operation of the system very intuitive. When the user tries to point out references that the agricultural robot is not able to reach, it activates its actuators by exerting pairs and forces that allow it to detect the event. For example, with this device the user can detect when trying to exceed the limit of $\pm 60^{\circ}$ of the joint 4 (one of the biggest limitations of the SR), every time that tries to exceed this range, activates the joint 4 of the MR exerting a force that forces the user to comply with the restriction, in addition to preventing the SR from colliding with itself, in addition it indicates the user the event and allows him to easily correct the reference. The Fig. 23 shows the MR with 5 degrees of freedom. 


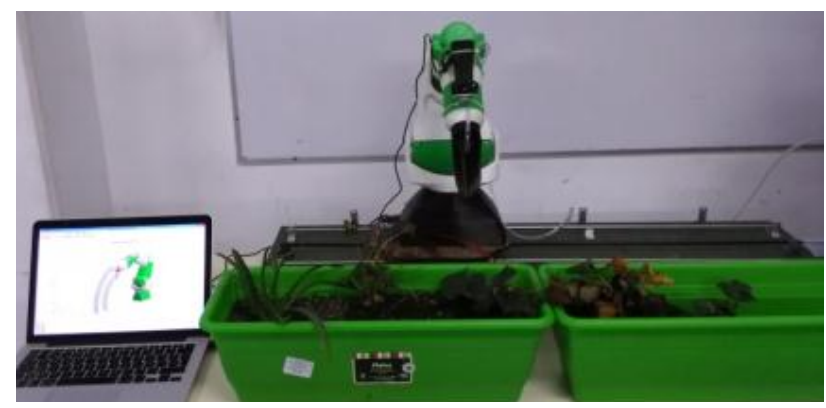

Fig. 24. Agricultural robot in the remote area of the teleoperation system.

To verify the operation of the teleoperated system, experiments were proposed where the user had to perform typical operations of an agricultural process such as planting, irrigation and fertilization of plants. In Fig. 24 a photograph of the SR and two pots where the plants that are under its care are housed.

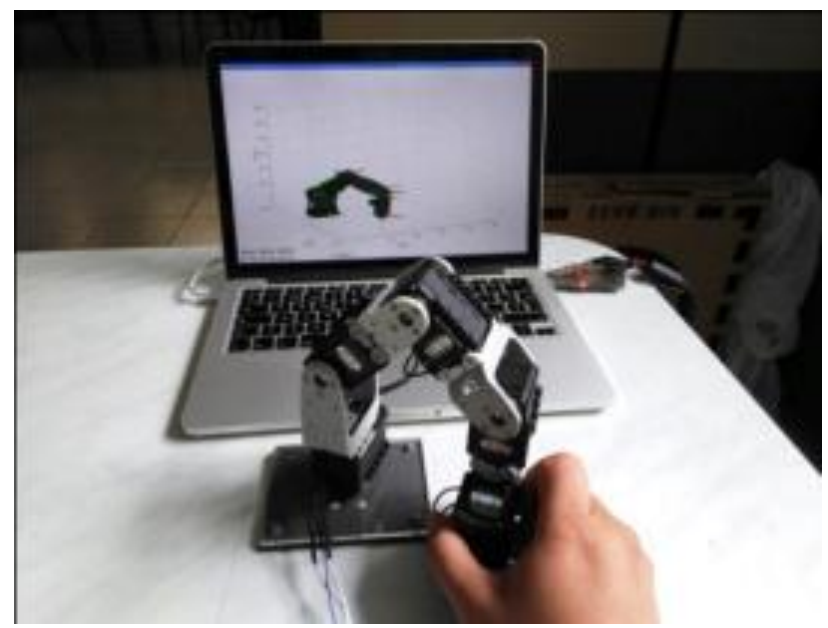

Fig. 25. User moving the low cost MR.

In Fig. 25 the user can be seen moving the MR to generate the references that are sent to the controller of the SR. The similarity between the SR and the MR can be clearly distinguished. It should be noted that the articulations of the MR are normally de-energized and only act when the user tries to make an erroneous movement that the robot in reality cannot do, avoiding collisions.



Fig. 26. Robot teleoperation interface.

To get better feedback of the environment, the user has access to an IP camera located in the remote area which allows him to clearly visualize the robot's actions and, if desired, the visual aids created for it, as shown in Fig. 26.
If the user wishes, the activation of the supply of information from the sensors is available, with this feature he can verify the temperature, humidity and luminosity of the crop so that he may be able to confirm that there is water or fertilizer in the storage tanks and carry out irrigation or fertilization tasks.

Teleoperation of the Robot Using Pre-Loaded Routines Mode: The teleoperation method based on the task space was designed to perform non-repetitive operations of a simple or medium degree of difficulty, for example the fumigation of a particular section of a plant. The teleoperation mode by means of the MR is planned for cases in which performing complex tasks that require greater skill of the user is desired, such tasks could be opening holes, planting plants or weeding. It should be noted that these activities were thought for the robot and not for a human since there is the possibility of having crops in places that are difficult to access such as vertical crops, roofs or terraces. In this way regular care can be provided to the crop without the user being subjected to dangerous situations (slippage, falls, among others).

In addition to the mentioned teleoperation methods, another mode has been generated to control the robot. This method is based on the execution of previously established routines which can be activated by the network sending high level commands via UDP. This method allows performing repetitive tasks such as irrigation and fertilization of plants or reading the information coming from the sensors from a mobile device (cellphones, tablets among others) or a computer. It must be taken into account that if the user wishes it, he can generate a repetitive schedule of tasks for the robot to execute based on or not based on criteria presented by the sensors. For example, water the plants every day at 5 a.m. except for the days when the temperature is lower than a predetermined value. In the same way conditions could be created according to luminosity and humidity, which can be very useful in countries with seasons.

\section{Results}

As for the design of the robot, it was possible to show that the torque calculation and the selection of the motors was correct considering that the robot achieves the preestablished speeds even in the most demanding configuration according to the gravitational effects. The functioning of the sensors is validated by comparison with the readings obtained by means of external equipment.

Agricultural robot joint level teleoperation results: To corroborate the functioning of the robot's teleoperation, a mono-articular study was used. Each of the joints was taken and their PID controllers were tuned (case MX64 motors) these are embedded in each of its drives. The control of each one of the drives is composed of two cascade systems which allow to define both the target position and the required speed.

Once the motors were calibrated, a comparative study was performed, emulating the position reference given by the articulation of the MR (step signal equivalent to a 30 degree offset) versus the response of the articulation of the SR implementing different speed levels. In Fig. 27 we can see the results corresponding to joint number 2 (the one that requires greater torque). These show the response to speeds ranging from 1 to 11 revolutions per minute (RPM). The 
results with higher speeds were not contemplated given that they gave a response with a peak value of over-oscillation which is not recommended in the case of teleoperated systems to avoid collisions. Since the idea is for the system is to respond as quickly as possible, it was selected to work with 11 RPM.

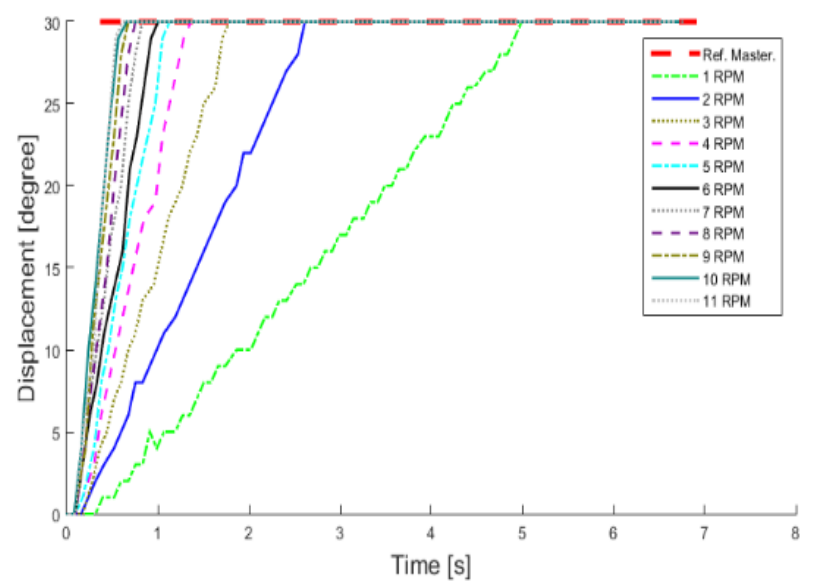

Fig. 27. Response of a joint of the SR against a step type reference of 30 degrees.

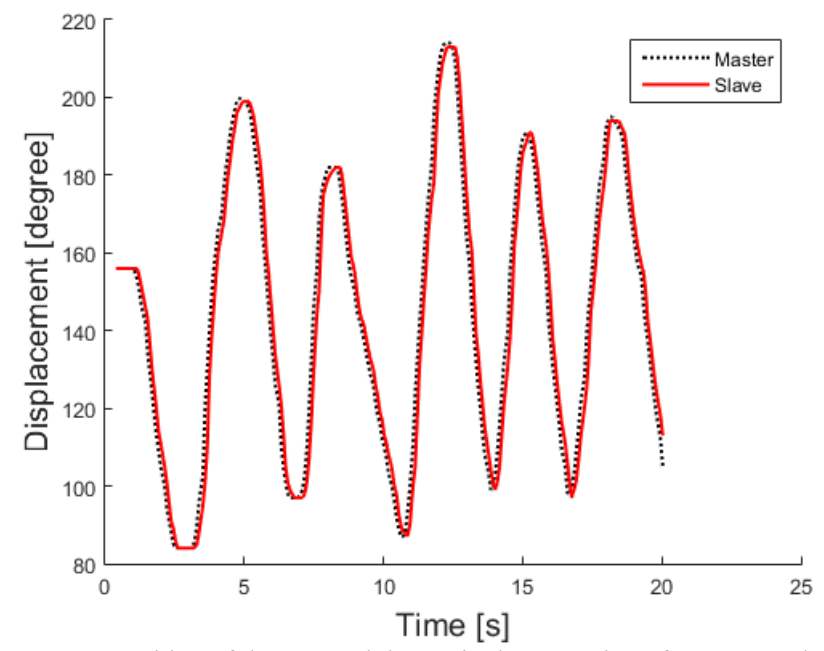

Fig. 28. Position of the MR and the SR in the execution of a 20 -second trajectory.

In Fig. 28 the results obtained for the same joint are illustrated, in an experiment where the user-directed MR was used executing relatively fast oscillatory movements for a time of 20 seconds. It can be seen how the articulation of the SR follows the reference adequately. It is necessary to emphasize that these experiments were carried out using a local network to guarantee low delays in the communications channel (provided for the tracking mode of the MR).

In order to quantify the performance of the system before delays (variables) occurred in the communication channel an experiment was proposed which emulates the position reference given by the articulation of the MR by a sinusoidal type signal with a unit frequency, a amplitude of 40 and a midpoint of 150 degrees. By sending the same reference, the evaluation of responses to different delays that occurred in the communications channel is allowed. To emulate the different levels of delay and to preserve the variable characteristics of the communications system, a buffer of variable amplitude ( 2 to 6 data) was created so that it delayed the sending of a data until the buffer was completely filled. It must be emphasized that non-blocking sockets were used via UDP to avoid possible inconveniences in the case of loss of information.



Fig. 29. Comparison of the tracking position of the SR with the same reference of sinusoidal type and different delays in the communications channel.

In Fig. 29, the response obtained with the same reference can be seen using a delay buffer of level 2 to 6 . It can be clearly seen that the system responds correctly despite the various variable delays.

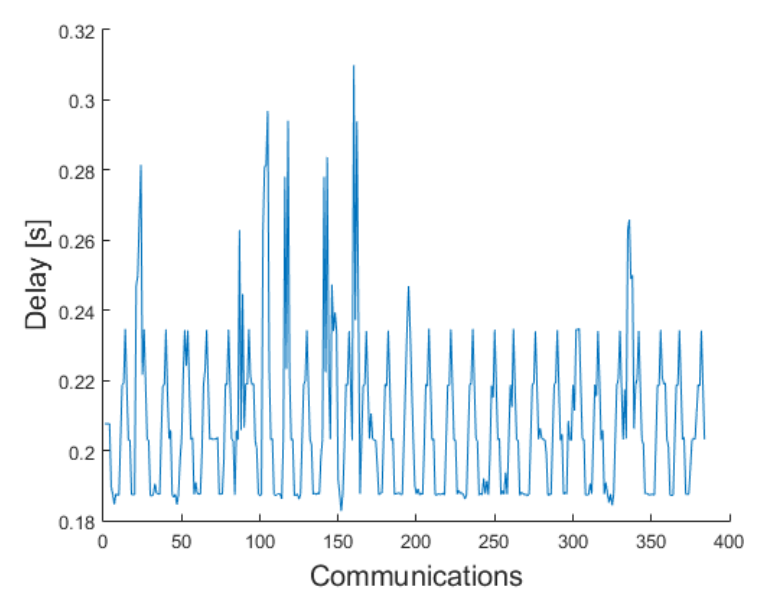

Fig. 30. Delay in the communication channel for the experiment called Slave R4.

In Fig. 30, the delays produced in all communications established for the experiment called Slave R4 (amplitude buffer 4) are illustrated, where the average value of the delays was 207, the maximum value of 31 and the standard deviation of 22 milliseconds

As is evident, the delay in the communications channel increases, the response developed by the articulation of the $\mathrm{SR}$ is more differentiated from that of the MR. In order to better parameterize the quality of the response developed, an identification of the response is employed using the form given by Eq. (6) and the error is verified with respect to it.

$$
q_{e}=40 \operatorname{sen}(t-\operatorname{lag})+150
$$

In this equation, the lag variable represents the estimated delay time that the articulation of the SR takes to perform the trajectory proposed by the MR; this time involves the 
delay in communications and the dynamics of the system and controller.



Fig. 31. Error in position of the SR with respect to the sinusoidal signal phase experiment called Slave R4.

In Fig. 31 the error obtained in position of the SR with respect to the offset sinusoidal signal ( 0.657 seconds) of the experiment called Slave R4 is presented.

Table 1. Metric of the experiments performed with different delays.

\begin{tabular}{|c|c|c|c|c|c|c|c|}
\hline \multirow{2}{*}{ 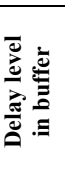 } & \multirow[b]{2}{*}{$\begin{array}{l}\text { Estim- } \\
\text { ated } \\
\text { Lag [s] }\end{array}$} & \multicolumn{3}{|c|}{$\begin{array}{c}\text { Communications Channel } \\
\text { delay [s] }\end{array}$} & \multicolumn{3}{|c|}{$\begin{array}{c}\text { Error regarding estimated } \\
\text { signal [degrees] }\end{array}$} \\
\hline & & $\begin{array}{l}\text { Avera- } \\
\text { ge }\end{array}$ & $\underset{\text { um }}{\operatorname{Maxim}}$ & STD & $\begin{array}{l}\text { Abso } \\
\text {-lute } \\
\text { ave- } \\
\text { rage } \\
\end{array}$ & $\begin{array}{c}\text { Absolu- } \\
\text { te maxi- } \\
\text { mum }\end{array}$ & STD \\
\hline 2 & 0.390 & 0.103 & 0.18 & 0.015 & 1.40 & 10.05 & 2.03 \\
\hline 3 & 0.517 & 0.159 & 0.29 & 0.035 & 1.55 & 8.98 & 2.09 \\
\hline 4 & 0.657 & 0.207 & 0.31 & 0.022 & 1.97 & 6.59 & 2.52 \\
\hline 5 & 0.797 & 0.258 & 0.37 & 0.037 & 2.56 & 8.42 & 3.27 \\
\hline 6 & 0.969 & 0.309 & 0.43 & 0.029 & 3.78 & 11.27 & 4.69 \\
\hline
\end{tabular}

Table 1 shows the metrics obtained for experiments where the buffer varies between 2 and 6 , which implies delays in the communications channel between approximately 100 and 300 milliseconds.

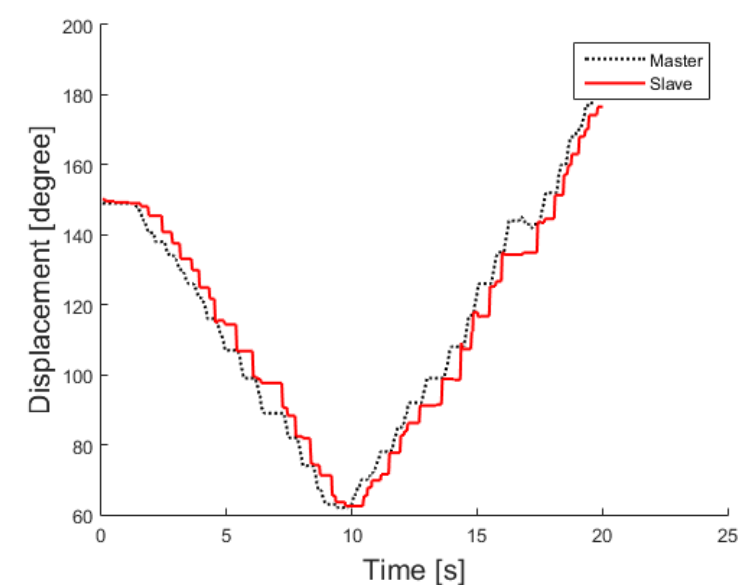

Fig. 32: Lock of the joints of the MR in face of a significant difference of positions regarding those of the SR or significant delays.

Additionally, if the user wishes to perform a task in which he must guarantee an error smaller than a certain value, he can activate a feature that enables a locking system of the joints of the MR to avoid significant differences between the MR and the SR, or in the event that large delays occur. Under normal conditions when the SR reaches the reference the joints are unlocked. In Fig. 32, an example of this type of actions is illustrated.

Descriptive results of the operation of the agricultural robot: Once the operation at the joint level of the agricultural robot is corroborated, tests in relation to the execution of the tasks for which it was designed were performed. For these tests the elements of the crop, the multipurpose effector, the displacement rail and the robot accessories were used.

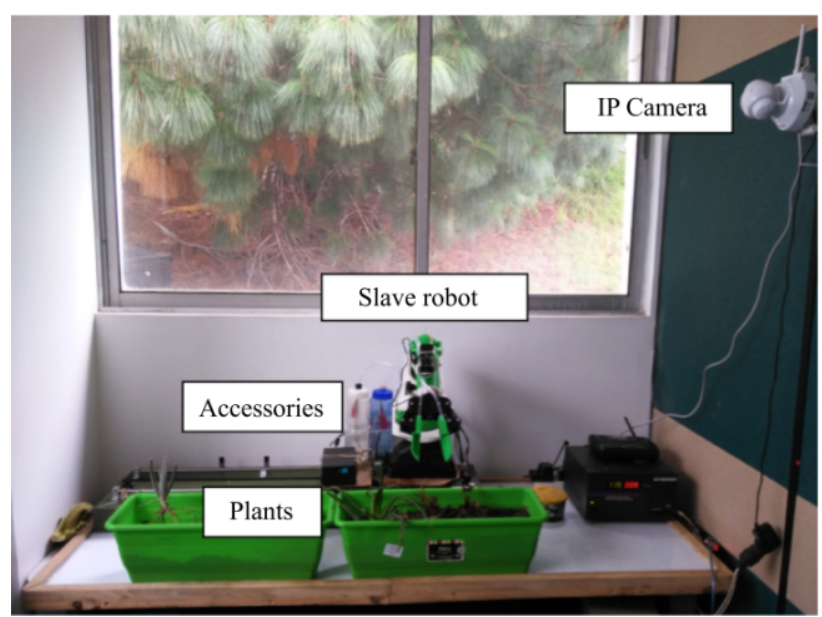

Fig. 33: Robot operation using guide signals of in the robot's workspace.

In Fig. 33 we present the teleoperation system corresponding to the remote area, where we can see cultivation, the SR with all accessories attached which, apart from the sensors, the use of an on-board camera that improves perception is highlighted. The user has access to an IP camera that allows a visual feedback of the environment.

In these tests, a user was asked to perform typical tasks that must be executed in the implementation of an urban crop. These tasks consisted in the teleoperation of the agricultural robot by means of the MR, which had the multipurpose manipulator on it. The tasks included the removal of a dry plant, the opening of a hole, and the sowing by transplant, in this case it can be observed that the plant is carried in a paper bag which degrades with time facilitating the planting process and lastly a cutting or pruning operation as it can be appreciated in Figs. 34 to 36.

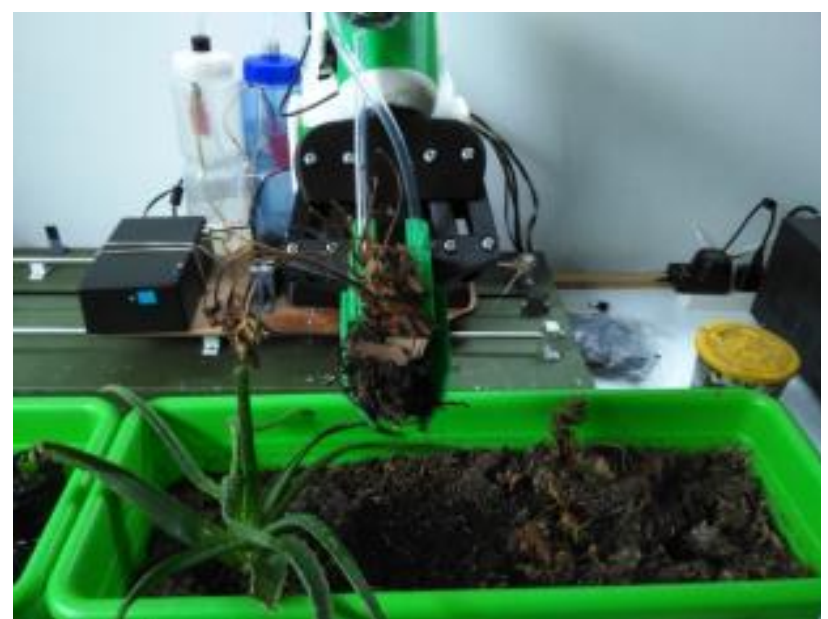

Fig. 34. Process of weeding or removal of plants.

As expected, the irrigation and fertilization operations were trivial, so they were destined to be executed in 
repetitive and continuous sequences through the use of mobile devices.

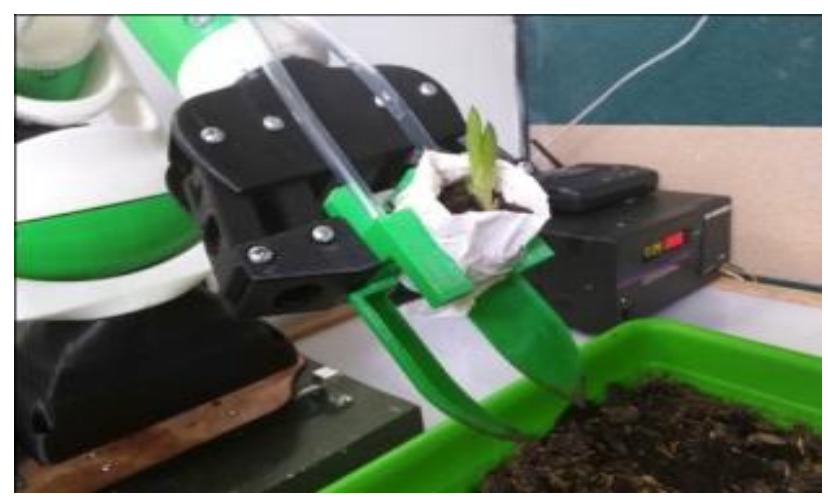

Fig. 35. Realization of the sowing process by transplant.

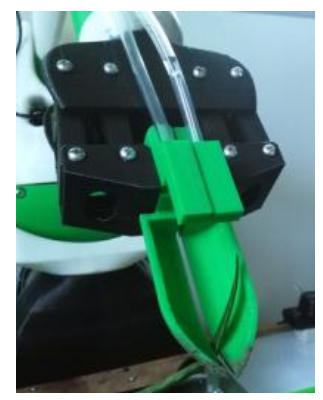

(a). Cut.

Fig. 36. Carrying out tasks.

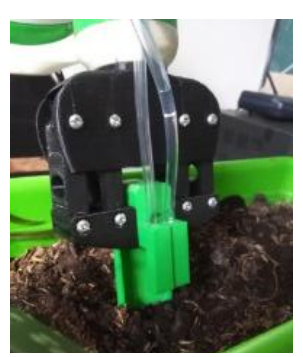

(b). Opening of holes.

\section{Conclusions}

With the execution of this project it can be concluded that it is viable to tend to a crop remotely in an urban environment. The teleoperation system is fundamental for the development of operations. Many variables must be taken into account to reduce dependence on supervision of the same. Within these variables, emphasize: system supplies such as electricity, water, fertilizer, soil, among others.

The sensors play a very important role in the teleoperation processes of this project, the cameras are transcendental since they allow to observe the behavior of the robot and the elements with which it interacts. On the other hand, the luminosity, temperature and humidity sensors can be used to generate optimal irrigation conditions according to the criteria established by the agronomist, as well as to avoid material waste and possible damage.

This is an Open Access article distributed under the terms of the Creative Commons Attribution License

\section{References}

1. Insu K., James, J., Crittenden, J., 2015. "The energy-efficient, economical, and environmental impacts of microturbines on residential customers," Green Technologies Conference (GreenTech), 2015 Seventh Annual IEEE., pp.139,146. DOI: 10.1109/GREENTECH.2015.20

2. Subbiah, K., Kannan, S., 2011. "The eco-friendly management of hotel industry," 2011 International Conference on Green Technology and Environmental Conservation (GTEC 2011), pp. 285,290. DOI: 10.1109/GTEC.2011.6167681

3. Aboul, E., Ibrahim, A., Abdel, A., Omar, A., 2014. "Developing of energy credits in an Egyptian Green Building Rating System," 2014 International Conference on Engineering and Technology (ICET), pp.1,6. DOI: 10.1109/ICEngTechnol.2014.7016768

4. Wei W., Cheng G., Xu S., Liang L., Lu G., Fu H., 2011. "Study on Water Requirements of Liuzhou Ecological City Construction," 2011 International Conference on Computer Distributed Control and Intelligent Environmental Monitoring (CDCIEM), pp.2248,2251. DOI 10.1109/CDCIEM.2011.241

5. Yie C., Chao L., Chih H., Yao, T., Hsueh C., 2009. "Applying GISbased rainwater harvesting design system in the water-energy conservation scheme for large cities," 13th International Conference on Computer Supported Cooperative Work in Design, CSCWD 2009, pp.722,727. DOI: 10.1109/CSCWD.2009.4968144

6. Xiangxiao M., 2013. "A brief analysis on the construction of urban greenway network in Zhongshan - Based on the perspective of environmental protection," 2013 6th International Conference on Information Management, Innovation Management and Industrial Engineering (ICIII), vol.2, pp.412,415. DOI: 10.1109/ICIII.2013.6703174

7. Xingzhou L., Yaowen X., Yuchu X., Chao Z., Xiangqian W., 2011. "Spatio-temporal processes and causes analysis of Jiayuguan oasis in China over a 23a period," 2011 19th International Conference on Geoinformatics, pp.1,4. DOI: 10.1109/GeoInformatics.2011.5980679

8. Plaskoff, R. (2014). "PhytoKinetic: Lightweight Green Roof System For City Buses and Vehicles, Urban Gardens", USA. http://www.urbangardensweb.com/2013/07/24/phyto-kinetic-greenroofs-for-city-buses-and-improved-urban-ecosystem/ (Consultado: 5 de agosto 2015)
9. Bechar, A., Vigneault, C., 2016. "Agricultural robots for field operations: Concepts and components”. Biosystems Engineering, 149, 94-111. http://doi.org/10.1016/j.biosystemseng.2016.06.014

10. Wei, X., Jia, K., Lan, J., Li, Y., Zeng, Y., \& Wang, C., 2014. "Automatic method of fruit object extraction under complex agricultural background for vision system of fruit picking robot". Optik, 125(19), 5684-5689. http://doi.org/10.1016/j.ijleo.2014.07.001

11. Ishibashi, M., Iida, M., Suguri, M., Masuda, R., 2013. "Remote monitoring of agricultural robot using web application". IFAC Proceedings Volumes Vol. 46. IFAC, http://doi.org/http://dx.doi.org/10.3182/20130828-2-SF-3019.00047

12. Han, L., Ruijuan, C., Enrong, M., 2016. "Design and simulation of a handling robot for bagged agricultural materials". IFACPapersOnLine, 49(16), http://doi.org/10.1016/j.ifacol.2016.10.032

13. Ryoo, Y. J., Lee, K. N., Choi, P. G., 2012. "Intelligent platform design of agricultural robot inspired by farmer assistance (AGRIFA)". 6th International Conference on Soft Computing and Intelligent Systems, and 13th International Symposium on Advanced Intelligence Systems, SCIS/ISIS 2012, 2037-2039. http://doi.org/10.1109/SCIS-ISIS.2012.6505371

14. Aronson, R., 2013. "FarmBot: Humanity's open-source automated precision farming machine", (678), 53. Retrieved from https://farmbot.io/whitepaper

15. Adamides, G., Katsanos, C., Parmet, Y., Christou, G., Xenos, M., Hadzilacos, T., Edan, Y., 2017. "HRI usability evaluation of interaction modes for a teleoperated agricultural robotic sprayer". Applied Ergonomics, 62, 237-246. http://doi.org/10.1016/j.apergo.2017.03.008

16. Amrita Sneha, A., Abirami, E., Ankita, A., Praveena, R., Srimeena, R., 2015. "Agricultural robot for automatic ploughing and seeding". Proceedings - 2015 IEEE International Conference on Technological Innovations in ICT for Agriculture and Rural Development, TIAR 2015, (Tiar), 17-23. http://doi.org/10.1109/TIAR.2015.7358525

17. Blender, T., Buchner, T., Fernandez, B., Pichlmaier, B., Schlegel, C., Thiemo, E., Benno, F., 2016. "Managing a mobile agricultural robot swarm for a seeding task", 0-7. http://doi.org/10.1109/IECON.2016.7793638 
18. Ando, H., Muramatsu, N., 2013. "Deformation constraint of elastic fingers of a monolithic gripping mechanism for enhancing handling range and applying to agricultural Robots", The SICE Annual Conference 2013, Nagoya, Japan, 2013, pp. 1606-1611. 2-7.

19. Rigatos, G. G., 2013. "Derivative-free distributed nonlinear Kalman filtering for cooperating agricultural robots". IEEE International $\begin{array}{llll}\text { Symposium on } & \text { Industrial }\end{array}$ http://doi.org/10.1109/ISIE.2013.6563603

20. Correll, N., Arechiga, N., Bolger, A., Bollini, M., Charrow, B., Clayton, A., Rus, D., 2010. "Indoor robot gardening: Design and implementation”. Intelligent Service Robotics, 3(4), 219-232. http://doi.org/10.1007/s11370-010-0076-1

21. Wang, M., Wang, X., Yi, X., Tu, J., 2012. "Experimental study on long-range navigation behavior of agricultural robots". Proceedings - 2012 International Conference on Computing, Measurement, Control and Sensor Network, CMCSN 2012, 409-412. http://doi.org/10.1109/CMCSN.2012.100

22. Rovira, F., Chatterjee, I., Siz-Rubio, V., 2015. "The role of GNSS in the navigation strategies of cost-effective agricultural robots". Computers and Electronics in Agriculture, 112, 172-183. http://doi.org/10.1016/j.compag.2014.12.017

23. English, A., Ross, P., Ball, D., Upcroft, B., Corke, P., 2015. "Learning crop models for vision-based guidance of agricultural robots". IEEE International Conference on Intelligent Robots and Systems, 2015-December, http://doi.org/10.1109/IROS.2015.7353516
24. Mahadhir, K. A., Tan, S. C., Low, C. Y., Dumitrescu, R., Amin, A. T. M., Jaffar, A., 2014. "Terrain classification for track-driven agricultural robots". Procedia Technology, 15, 775-782. http://doi.org/http://dx.doi.org/10.1016/j.protcy.2014.09.050

25. Levin, M., Degani, A., 2016. "Design of a task-based modular reconfigurable agricultural robot". IFAC-PapersOnLine, 49(16), 184189. http://doi.org/10.1016/j.ifacol.2016.10.034

26. Baur, J., Pfaff, J., Ulbrich, H., Villgrattner, T., 2012. "Design and development of a redundant modular multipurpose agricultural manipulator". IEEE/ASME International Conference on Advanced Intelligent Mechatronics, AIM, 823-830. http://doi.org/10.1109/AIM.2012.6265928

27. Akao, Y., 2004. "Hoshin Kanri: Policy deployment for successful TQM". SteinerBooks.

28. Kume, H., Vasco, E., 1992. "Herramientas estadísticas básicas para el mejoramiento de la calidad". Editorial Norma.

29. Jazar, R., 2010. "Theory of applied robotics: kinematics, dynamics, and control". (Springer, Ed.) (2nd ed.). Melbourne. http://doi.org/10.1007/978-1-4419-1750-8

30. Cheng, F., Hour, T., Sun, Y., Kung, F., 1995. "Analysis and resolution of singularities for 5-DOF Gryphon manipulator". In IEEE International Conference on Systems, Man and Cybernetic Vol. 5, pp. 4416-4421. http://doi.org/10.1109/ICSMC.1995.538489

31. García, F., 2015. Tesis: Desarrollo de dispositivos de apoyo para el proyecto cultivo robótico remoto. Universidad de Pamplona. 